\title{
Fabrication and characterization of two-dimensional photonic crystal using holographic lithography technology
}

\author{
Kuo-Chang Lo, Yi-Sheng Lan, Mei-Li Hsieh* \\ Institute of Electro-optical Science and Technology, National Taiwan Normal University, Taipei, Taiwan \\ mlh@ntnu.edu.tw
}

\begin{abstract}
We propose and demonstrate a holographic system using three beams to generate periodic patterns for fabricating photonic crystals. By properly adjusting the incident angle and intensity of three laser beams, the period and structure of interference pattern can be modified. Computer simulation results will be presented and optical holographic system will be constructed. In addition, a simple optical inspection method for real-time examining the structure of photonic crystal will be developed. Optical experimental result will be demonstrated and discussed in this paper.
\end{abstract}

Keywords: Holography, photonic crystal.

\section{Introduction}

Due to the attractive properties of the photonic crystals, it has been widely studied and applied on interesting areas in resent years [1], such as optical waveguide, photonic integration circuits, optical component, high extraction rate LED, and so on. However, there are several techniques to fabricate the photonic crystals; for example, photolithography in IC foundry, electronic beam lithography, nano-imprinting and holographic lithography...etc [2-4]. Among those fabricating technique, one of particular feature of the holographic lithography is able to produce 1-D, 2-D or 3 -D photonic crystal by one step exposure in a large area without complicate manufacture process which can meets the demand of mass production. Therefore, in this contribution, we design a holographic system with three laser beams to generate the structure of photonic crystals. Computer simulation results will be presented, as effect of intensities and incident angles of three laser beams. By using our holographic system, the designed photonic crystals can be fabricated and the optical experimental results also will be demonstrated in this paper.

* corresponding author

\section{Design of holographic system}

The interference pattern generated by multi-beams is periodic and analogous with the ordered structure of photonic crystals. When the photoresist is exposed by the interference pattern, the photonic crystal structure can be fabricated after development and lithography procedures. Therefore, this technology is so-called holographic lithography or interference lithography. Hence, we design a holographic system with three beams, which interfere by the same incident angle between each two neighboring beams. The schematic diagram of our holographic system is shown in figure 1. By using this system, the interference pattern can be generated as the triangular periodic structure. In this case, the photonic crystals with this structure have high possibility to form complete photonic band gap [5]. The intensity distribution of the interference pattern can be written as [6]:

$I(x, y, z)=\sum_{j} E_{j}^{2}+\sum_{i<j} 2 E_{i} E_{j} \cos \alpha_{i j} \times \cos \left[\left(\vec{K}_{i}-\vec{K}_{j}\right) \cdot \vec{r}+\delta_{i}-\delta_{j}\right]$,

$\mathrm{i}, \mathrm{j}=1,2,3$

where $E, \vec{K}$ and $\delta$ represent the electric field, wave vector and initial phase of laser beams, respectively. The footnote $i, j$ denotes the number of incident beams. The parameter $\alpha_{i j}$ is the angle between polarization orientation of each two incident beams. To simplify the simulation model, we assume the incident beams have the same polarization orientation, i.e. $\alpha_{\mathrm{ij}}$ will be zero. According to the equation (1), we can obtain the intensity distribution of the interference pattern in figure 2. The period $d$ of the interference pattern is a function of the incident angle between each two neighboring beams as:

$d=\lambda /[\sqrt{3} \sin (\theta / 2)]$

where $\lambda$ is the wavelength of laser beam. Hence, the period $d$ will be decreasing with increasing the incident angle $\theta$ and decreasing the wavelength $\lambda$. The curve of the relationship between period and the incident angle is plotted in figure 2 with $\lambda=355 \mathrm{~nm}$. 
In this figure, it shows the minimum period with $\lambda=$ $355 \mathrm{~nm}$ is $236 \mathrm{~nm}$. We can properly adjust the incident angle of laser beams to obtain the period $d$ as our expectation.

In addition, the intensities of the incident beams will affect the shape of bright region in interference pattern. When the intensity of each laser beams is equal, the shape of the bright region is a circular spot, which is shown in figure 3(a). However, if the intensity ratio of laser beams is modified as $I_{1}: I_{2}: I_{3}=1: 1: 4$, the shape of bright region becomes an elliptical spot and the major axis of the ellipse is along the incident direction with the strongest intensity. The computer simulation result is shown in figure 3(b). Consequently, the interference pattern with different intensity ratios also are calculated and shown in figure 3(c) and 3(d). According to figure 3, we can modify the shape the bright region in the interference pattern by adjusting the intensity ratio of the incident beams.

\section{Optical experiments}

The architecture of our holographic system is shown in figure 4. The expanded laser beam passes through two beam splitters, and is divided into three beams. Each beam is reflected by mirror with appropriate arrangement. Then, all the three beams intersect symmetrically at the same position, where the photoresist is located. Therefore, the incident angles of the laser beams can be modified by adjusting the reflected angle from mirrors $\mathrm{M}_{1}, \mathrm{M}_{2}$ and $\mathrm{M}_{3}$. The triangular periodic pattern can be obtained by our holographic system, and the period is dependent on the incident angles as the simulated analysis in figure 2 .

Here, the wavelength of laser beam is $355 \mathrm{~nm}$, for example, the incident angle between each two neighboring beams is about $28^{\circ}$. Therefore, the period of the interference pattern is about $430 \mathrm{~nm}$. In addition, the intensities of three beams are the same, and the polarization orientations are along the same direction. On the other hand, the photoresist we used are AZ TFP 5F and AZ $150038 \mathrm{cp}$, which was spin on the glass. The thickness of the photoresist are about $0.6 \mu$ $\mathrm{m}$ and $2 \mu \mathrm{m}$ respectively, and the sample is put in the intersection of three beams. After exposure, the photonic crystal structure can be implemented in figure 5(a). It shows that the period, which is about $420 \mathrm{~nm}$, which is consistent with theoretical simulation. Figure 5(b) shows the other experimental result with the incident angle is about $20.43^{\circ}$. The period of the photonic crystals is about $587 \mathrm{~nm}$. Therefore, we can fabricate the photonic crystal with the different period using our holographic system.

\section{Optical inspection method}

In general, for inspecting the structure of photonic crystal, it should be observed by high precision instruments as atomic force microscope (AFM) or scanning electron microscope (SEM). However, these equipments can not buy easily due to the high price, and can not obtain the results in real-time due to the complicated operation procedure. Therefore, we propose a simple optical inspection method to realtime characterize the structure of photonic crystal during the exposure procedure.

Actually, a 2-D photonic crystal structure can be considered as a 2-D grating, as shown in figure 2 . The transmission coefficient of photonic crystal structure is written as [7]:

$P(x, y)=\left[\operatorname{comb}\left(a_{1} x+b_{1} y, a_{2} x+b_{2} y\right)\right] \otimes \operatorname{circ}(r)$

where $a_{1} / b_{1}=-\sqrt{3} / 3, a_{2} / b_{2}=\sqrt{3}$ in our case. If we use a plane wave to illuminate the 2-D photonic crystal, the Fraunhofer diffraction pattern can be observed in the far-field region according to the diffraction theory [8]. The intensity distribution of Faunhofer diffraction can be written as:

$$
\begin{aligned}
& O\left(f_{x}, f_{y}\right)=F\left[\operatorname{comb}\left(a_{1} x+b_{1} y, a_{2} x+b_{2} \mathrm{y}\right)\right] F[\operatorname{circ}(r)] \\
& \quad=|D|^{-1} \operatorname{comb}\left(b_{2} f_{x} / D-a_{2} f_{y} / \mathrm{D},-b_{1} f_{x} / \mathrm{D}+a_{1} f_{y} / \mathrm{D}\right) \mathrm{J}_{1}(2 \pi \rho) / \rho
\end{aligned}
$$

where $D=a_{1} b_{2}-a_{2} b_{1}, r$ is radius of bright spot. $f_{x}, f_{y}$ and $\rho$ represent spatial frequencies. Compare the equation (3) and (4), it shows that the diffraction pattern of the photonic crystal has similar configuration with the photonic crystal structure. The intensity distribution of the diffraction pattern is presented by triangular structure. The period of the diffraction pattern is a function of the period of the photonic crystal and the distance $\mathrm{z}$ between the photonic crystal and the observed plane. However, the diffracted angle $\phi$ is defined as the ratio of the period of the diffraction pattern to the distance z. Therefore, the diffracted angle is dependent on the period of the photonic crystal, and the relationship can be written as [9]: $\quad d \sin \phi=\lambda_{t}$

where $\lambda_{t}$ is wavelength of the illuminating light. For a given wavelength $\lambda_{t}=355 \mathrm{~nm}$, and the relationship between the diffracted angle and the period of the photonic crystal can be plotted as figure 6. It represents that the period of photonic crystal can be evaluated by measuring the diffracted angle. For example in the case (c) of figure 6, the diffracted angle can be measured from the diffracted pattern in figure $6(c)$ and is about $36.3^{\circ}$. According to equation (5), we can calculated the period of the photonic crystal is about $0.6 \mu \mathrm{m}$. By using SEM instrument, we also can obtain the period of the photonic crystal is about 0.6 $\mu \mathrm{m}$ as shown in figure $5(\mathrm{~b})$. It shows the consistent result by using two different methods. Therefore, the simple inspection method can easily characterize the structure of the photonic crystal and also can observe 
the fabricating procedure of the photonic crystal during the exposure in real-time.

\section{Conclusion}

In this paper, we demonstrated a holographic system to fabricate photonic crystal. The computer simulations have been discussed. The structures of photonic crystals have been fabricated by using our holographic system. The minimum period in our system which now can be achieved is about $430 \mathrm{~nm}$. In addition, a simple inspection method has been proposed and presented in this paper.

\section{Acknowledgements}

This work was supported by a grand from National Science Council of R.O.C. Taiwan, under contract no. NSC 94-2622-E-003-004-CC3 and NSC 94-2215-E003-001.

\section{Reference}

[1] E. Ozbay, I. Bulu, K. Aydin, H. Caglayan and K. Guven, "Physics and applications of photonic crystals," Photonics and nanostructuresfundamentals and applications, 2, pp. 87-95, 2004..

[2] M. Campbell, D.N. Sharp, M.T. Harrison, R.G. Denning and A.J. Turberfield, "Fabrication of photonic crystals for the visible spectrum by holographic lithography," Nature, vol. 404, pp.53-56, 2000.

[3] A. David et al., "Photonic bands in two dimensionally patterned multimode $\mathrm{GaN}$ waveguides for light extraction," Applied Physics letters, vol. 87, 101107, 2005.

[4] A. Yokoo et al., "Direct nanoprinting technology and its application to nanostructure fabrication," IEEE LEOS $13^{\text {th }}$ annual meeting, vol.2, pp. 417418, Nov. 13-16 2000.

[5] K. Inoue and K. Ohtaka, Photonic crystals physics, fabrication and applications, Springer, 2004.

[6] L. Z. Cai, X. L. Yang, and Y. R. Wang, "All fourteen Bravais lattices can be formed by interference of four noncoplanar beams," Optics Letters, vol. 27, pp. 900-902, 2002.

[7] J.D. Gaskill, Linear systems, Fourier Transforms, and Optics, John Wiley \& Sons, New York, pp. 77-95, 1978.

[8] J. Goodman, Introduction to Fourier optics $2^{\text {nd }}$, McGrawHill, 1996.

[9] E. Hecht, Optic $4^{\text {th }}$, Addison Wesley, pp. 477, 2002.

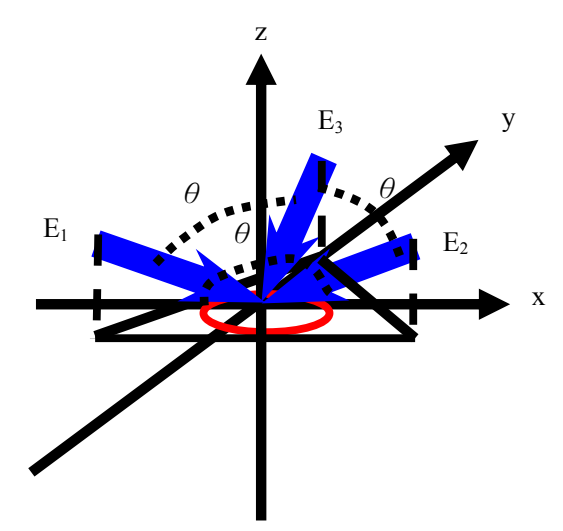

Figure 1 The schematic diagram of holographic system with three beams.

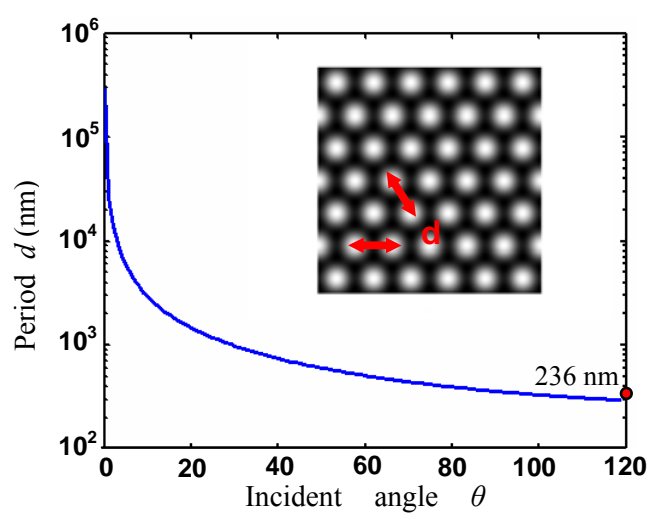

Figure 2 The period of the interference pattern as a function of the incident angle.

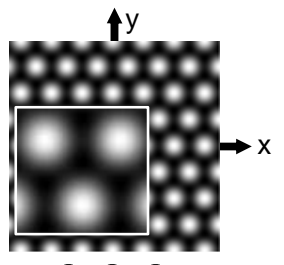

$\mathrm{I}_{1}=\mathrm{I}_{2}=\mathrm{I}_{3}$

(a)

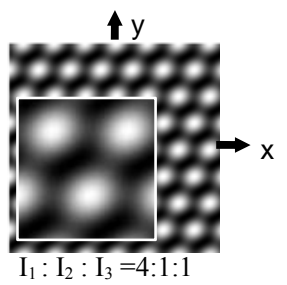

(c)

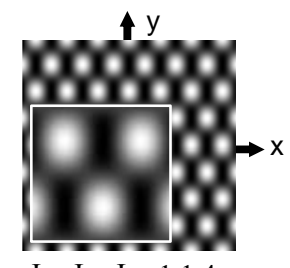

(b)

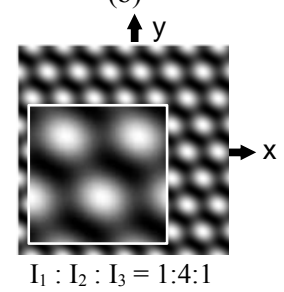

(d)
Figure 3 The intensity distribution of the interference pattern as a function of the beam ratio of the incident beams. 


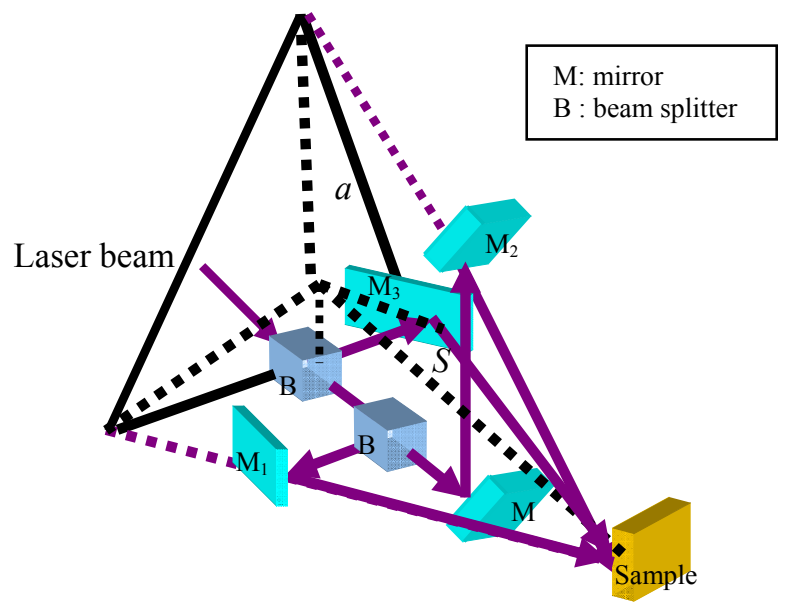

Figure 4 The architecture of out holography system.

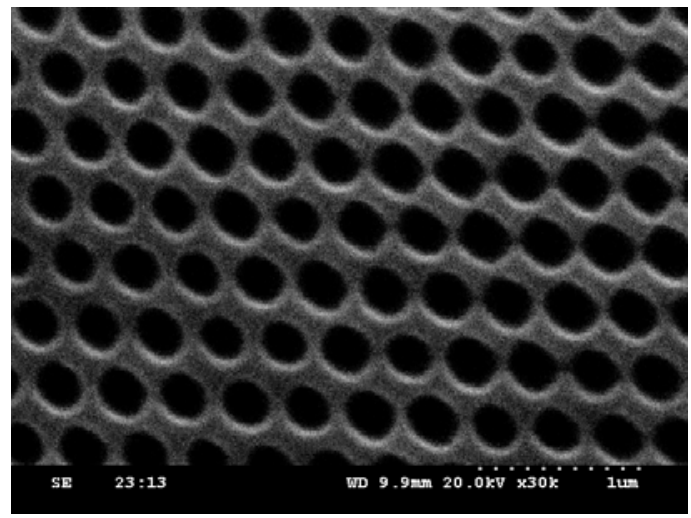

(a)

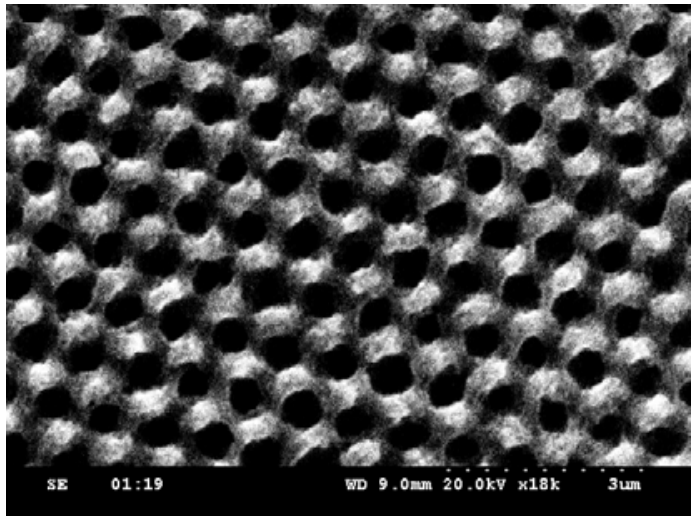

(b)

Figure 5 SEM pictures of our experimental results. The designed period is $430 \mathrm{~nm}$ in (a) and $587 \mathrm{~nm}$ in (b), measured period is about $420 \mathrm{~nm}$ in (a) and 600nm in (b).

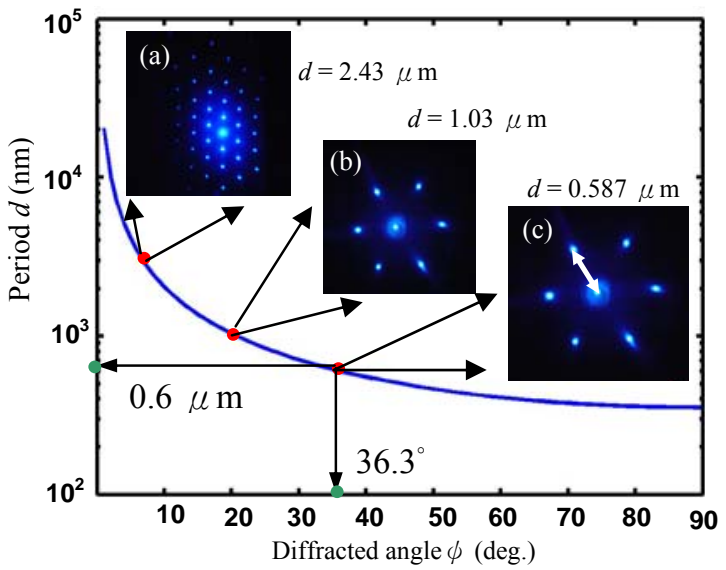

Figure 6 The period of the photonic crystal as a function of the as diffracted angle. 